CLINICAL GOVERNANCE

\title{
Clinical governance for better sexual health services
}

Gill Wakley, MD, MFFP, Visiting Professor in Primary Care Development, Staffordshire University, Stafford, UK and Freelance General Practitioner

Correspondence: Professor Gill Wakley.E-mail: gillwak@aol.com

(Accepted 15 July 2004)

Journal of Family Planning and Reproductive Health Care 2004; 30(4): 260-262

\section{What is clinical governance for?}

Providing sexual health services of high quality, with guaranteed minimum standards of care for all users at all times, is a major challenge. Variation in the quality of sexual health services is common. Standards vary in different units or practices in the same locality, between staff of the same discipline working in the same area, and between the care given to some sections of the population as compared to others. Clinical governance offers a co-ordinated approach to overcoming areas of risk or inequality through a combination of clinical and organisational improvements to the quality of health care practice.

\section{The elements of clinical governance}

In Making Clinical Governance Work for You, ${ }^{1}$ Ruth Chambers and I identified the following 14 themes as core components of professional and service development which taken together constitute clinical governance. These are:

1. Learning culture: in every department.

2. Research and development culture: throughout the National Health Service (NHS).

3. Reliable data: recorded once, readily available but secure.

4. Well-managed resources and services: in conjunction with social care and local authorities and available to all.

5. Coherent team: within your workplace or Trust.

6. Meaningful involvement of patients and the public: in your workplace, Trust and the NHS.

7. Health gain: activities to improve the health of patients served by your team and throughout different geographical areas of the NHS.

8. Confidentiality: of information in consultations, in medical notes, between practitioners.

9. Evidence-based practice and policy: applying it in clinical work, in the Trust, across the NHS.

10. Accountability and performance: for standards, performance of individuals, the work team, the management and the NHS - to the public and those in authority.

11. Core requirements: a good fit between skill mix and competence, communication, workforce numbers, morale in the workplace and across the Trust.

12. Health promotion: for patients, the public - targeting those with most needs.

13. Audit and evaluation: of the performance or achievements of the individual, the team and district services.

14. Risk management: proactive review, follow-up, risk management and risk reduction.

The components of clinical governance are not new. Bringing them together under the banner of clinical governance and introducing explicit accountability for performance is a new style of working. Carefully evaluating your own work, and that of your service, then making improvements in patient care, will enable you to form your own view about the place of clinical governance.
What does the government want?

The aims of the government's programme of modernisation of the health services are to: ${ }^{2}$

- Tackle the causes of ill health

- Make services convenient, quick and easy to use

- Ensure the consistency of services regardless of where you live

- Try and provide 'joined up' services that are not constrained by artificial barriers between services, such as health and social services

- Invest in improving the workforce and infrastructure.

The government sees this happening through: ${ }^{3}$

- Clear national standards set by the National Service Frameworks (NSFs) and National Institute of Clinical Excellence (NICE)

- Local delivery of quality services

- Monitoring of services through the Health Care Commission

- Consultation with patients and the public.

The recently introduced quality and outcome frameworks (QOFs) will provide one way of demonstrating what standards individuals and services are achieving. However, the clinical areas covered are limited at present and most of the quality and outcomes data for sexual health services will be about additional services, holistic care and access. If you are involved in commissioning or providing services, you will have been deluged with information that is in rapid development. ${ }^{4}$

\section{Management requirements}

Primary care organisations, hospital trusts and strategic health authorities are required to report on clinical governance each year. The Department of Health published a document to harmonise the requirements for reporting on clinical governance. 5 The advice was developed in consultation with the Commission for Health Improvement (now the Healthcare Commission), the National Clinical Governance Support Team (CGST) and the regional office clinical governance leads. It was tested with (then newly constituted) strategic health authorities. To meet the requirements of all these bodies you may be completing information in a formalised framework as follows:

- The patient's experience including:

- the planning and organisation of care

- the environment of care.

- The use of information including:

- information about the patient's experience

- information about resources and processes

- information about the outcomes of patient care.

- The processes for quality improvement including:

- risk management processes

- clinical audit programmes

- evidence-based practice and clinical effectiveness programmes

- learning from incident reporting

- learning from complaints. 
- Staff focus including:

- staffing and staff management

- education, training and continuous personal development

- multidisciplinary team working.

- Leadership, strategy and planning including:

- consultation and patient involvement

- organisation and clinical leadership

- planning of services

- organisational performance review

- health community partnerships.

Familiarity with the headings will help you to produce your own record keeping in a form that is easily translated for management use.

\section{Clinical governance in practice}

In the Sexual Health and HIV Commissioning Toolkit for Primary Care Trusts and Local Authorities, ${ }^{6}$ Appendix 8 gives an example of a clinical governance framework for professionals providing sexual health services. The framework is based on one primary care clinical governance model and is divided into four quadrants, making the interlinking parts more manageable. A sexual health needs assessment underlies all the provision of sexual health services. Without knowing what is needed, you cannot begin to evaluate what progress you are making towards meeting those needs. 7,8

Quality assurance measures

You must have quality assurance measures to maintain standards in all the quadrants below. These include confidentiality, appropriateness, availability, continuity, coordination, safety, respect, caring and timeliness.

\section{The first quadrant}

The first quadrant in the model ${ }^{6}$ concerns the range of clinical services or tasks (Box 1). The tasks described are for a generalist professional providing a holistic sexual health service. The balance of the services provided depends on the nature of the service and the needs of the clients seen. This should have been determined by the sexual health needs assessment.

Both employers and employees have a duty to ensure that health professionals and other staff have appropriate training and qualifications and that these are kept up to date. Quality assurance monitoring should develop a needs-led, evidence-based and consistent approach. Although such monitoring is largely the responsibility of

Box 1: The possible range of clinical sexual health services

- History taking, examination, diagnosis and treatment

- Diagnostic tests, including for pregnancy and infection

- Referral to other agencies

- Screening tests

- Counselling

- Prescribing and dispensing

- Undertaking procedures or operations such as long-term methods of contraception

- Follow-up and ongoing support

- Partner notification of sexually transmitted infections

- Team approach so that skills are used wisely

- Health promotion

- Participation in continuous professional development

- Provision of training

- Keeping patient records and data collection

- Undertaking audit and research
Box 2: Possible external links with sexual health services

- Maternity and gynaecology services

- Pathology and radiology services

- General medicine, paediatrics and dermatology

- GPs, health visitors, school nurses, dentists, pharmacists, dieticians and other professionals allied to medicine

- Youth and community services

- Child protection services

- Social services

- Schools and colleges

- Drug agencies

- HIV services

- Brook, Family Planning Association, Marie Stopes and other related organisations

- Benefits Agency

- Police

- Public health

- Health promotion

individual health professionals and their teams, any shortfall identified should be made known to the managers of the organisation.

\section{The second quadrant}

Health professionals need to establish external links in order to carry out their clinical work (Box 2). The links

Box 3: Organisational support for sexual health clinical services

- Clerical and administrative support (e.g. enough staff to answer the telephone promptly and suitably trained to answer queries correctly, experienced staff to run the service)

- Information technology (e.g. to make appointments easily, keep records confidential and yet accessible when needed, provide lookup facilities for information not readily at hand, help to monitor standards)

- Site location and facilities appropriate to the task (e.g. provide welcoming and easily accessible premises open at convenient times for clients)

- Appropriate equipment, supplies and storage space (e.g. to be able to provide the clinical service required at the time)

- Appropriate caseload or workload (e.g. the staff are not too busy to listen to what people need)

- Efficient patient record system (e.g. one able to record information once, accurately and reliably and make it available when required)

- Pathology collection service (e.g. so that tests can be done in a timely way and as accurately as possible)

- Sterilised supplies or sterilisation procedures (e.g. so that procedures can be done safely)

- Published service standards are monitored (e.g. to demonstrate those standards are being reached)

- Security at the workplace (e.g. clients and staff feel safe and not threatened in any way)

- Recruitment and retention policies for appropriately qualified staff (e.g. people know what they are doing and give a good service that is recognised by others)

- Robust recruitment arrangements for those working with young people (e.g. not just checked for safety but with the right attitudes)

- Human resources and employment policies supportive of part-time and multi-site working (e.g. staff who know and appreciate the difficulties)

- Financial support and time to enable professional and personal development (e.g. staff who have time to keep up-to-date and healthy)

- User consultation and robust complaints procedures (e.g. to try to prevent risk and put right those things that go wrong)

- Service promotion and advertising (e.g. so that users know what is available and where) 
Box 4: External contexts for sexual health services

- Community profiles and health needs assessments

- Local plans including the teenage prevention of pregnancy plan

- Government strategies (e.g. teenage pregnancy ${ }^{9}$ and National Strategy for Sexual Health and HIV ${ }^{10}$ )

- Fraser guidelines on advising and treating young people

- Venereal disease regulations and other public health law 11

- Governmental publications such as Organisation with a Memory, Data Protection Acts 1984 and 1988, Caldicott guidelines on confidentiality and the use of data, Access to Medical Records Act, Crown guidelines on nurse prescribing, etc. ${ }^{2,12}$

- Medicine Control Agency guidelines ${ }^{13}$

- Abortion Act ${ }^{11}$

- Child Protection procedures ${ }^{11}$

- Nursing and Midwifery Council (NMC) and General Medical Council (GMC) guidance

- Advice from medical and nursing colleges

- National Institute for Clinical Excellence (NICE)

- Health and safety regulations ${ }^{11}$

- National voluntary organisations ${ }^{14}$

have to be recognised and promoted by the organisation. A sexual health needs assessment identifies many of the organisations or individuals that are already providing services or whose services require development in some way. In some services, some of the links may be internal and provided within the service or alongside the sexual health service.

\section{The third quadrant}

This section lists the organisational support that health professionals require when undertaking clinical work. The organisation's management is responsible for ensuring that this essential non-clinical support is provided. A sexual health needs assessment will inform these support services and you will be able to add other examples of requirements in your own local area (Box 3).

The fourth quadrant

A sexual health needs assessment will identify many community and district health needs. The national and local policy contexts need to be taken into account. These external influences (Box 4) affect the way in which service provision is managed.

\section{Summary}

A clinical governance framework provides the tools to work towards ensuring that the sexual health service that you provide:

- Discovers and meets the needs of individual patients

- Improves the health of the public and reduces inequalities in access, services or treatment

- Demonstrates clinical quality (doing the right thing at the right time to the right people)

- Provides clinical accountability through audit and evaluation

- Includes multidisciplinary learning and development

- Supports open and reflective practice

- Respects and supports colleagues

- Works in partnership with others.

If you are ignorant of where you are now, you cannot make progress towards your goal.

Statements on funding and competing interests

Funding. None identified.

Competing interests. None identified.

References

1 Chambers R, Wakley G. Making Clinical Governance Work for You. Oxford, UK: Radcliffe Medical Press, 2000.

2 Department of Health. A First Class Service: Quality in the New NHS. Health Service Circular: HSC (98)113. London, UK: Department of Health, 1998.

3 Department of Health. The New NHS: Modern, Dependable. London, UK: The Stationery Office, 1997.

$4 \mathrm{http} / / / \mathrm{www}$. natpact.nhs.uk/primarycarecontracting/16.php.

5 http://www.dh.gov.uk/assetRoot/04/03/27/83/04032783.pdf

6 Department of Health. Effective Commissioning of Sexual Health and HIV Services - A Sexual Health and HIV Commissioning Toolkit for Primary Care Trusts and Local Authorities. London, UK: Department of Health, 2003

7 Wakley G, Cunnion M, Chambers R. Improving Sexual Health Advice. Oxford, UK: Radcliffe Medical Press, 2003.

8 Smith A. Young people's contraception and sexual health: report of a local needs assessment in Staveley, North Derbyshire. J Fam Plann Reprod Health Care 2001; 27: 29-32.

9 Social Exclusion Unit. Teenage Pregnancy. London, UK: The Stationery Office, 1999.

10 Department of Health. National Strategy for Sexual Health and HIV. London, UK: Department of Health, 2001.

11 Beresford N, Branthwaite M. Law for Doctors. London, UK: Royal Society of Medicine Press Ltd, 2003.

12 http://www.dh.gov.uk.

13 http://www.mca.gov.uk.

14 http://www.ncvo-vol.org.uk.

\section{BOOK REVIEW}

Obstetric and Gynecologic Emergencies: Diagnosis and Management. M Pearlman, J Tintinalli and P Dyne. New York, NY: McGrawHill, 2004. ISBN: 007137937 1. Price: $£ 55.00$. Pages: 566 (paperback)

With the current trend towards the centralisation of maternity services, it is likely that ultimately many pregnant women may only have immediate access to their local accident and emergency department. It is perhaps timely that this text (albeit written from the American viewpoint) addresses obstetric and gynaecological problems from the casualty gynaecological pro
officer's perspective.

The book is a well-illustrated, comprehensive reference guide to the management of urgent medical problems occurring in women. Chapters are organised and written with the main purpose of guiding the clinician quickly from presenting signs and symptoms to a differential diagnosis. It is easy to read and includes a bulleted list of key facts at the start of each chapter and highlighted diagnostic and treatment guidelines.

The obstetric section includes the immediate management of ectopic pregnancy, pre-eclampsia, diabetes, preterm labour, abdominal pain and surgical complications and the management of trauma. The chapter and the management of trauma. The chapter
on drug use in pregnancy includes many useful websites on this topic. There is also an interesting chapter summarising considerations when transferring pregnan women, as well as a valuable and detaied section on cardiopulmonary resuscitation and emergency perimortem Caesarean delivery. Unfortunately, the chapters on vagina bleeding and the complications of induced abortion are less appropriate for the UK-based practitioner. The American use of anti-D does not follow the RCOG guidelines and medical management of first-trimester abortions follows a different protocol to that used in the UK. Furthermore, due to the prevalence of UK. Furthermore, due to the prevalence of
surgical above medical management for midtrimester abortions and the use of paracervica blocks to evacuate the uterus in the USA, a quite different perspective on the UK management of these cases could easily be conveyed to the non-obstetrically trained casualty officer.

Coverage in the gynaecology chapters includes a helpful paediatric and adolescent section on the best techniques for pelvic examination, as well as the management of cases of sexual assault and abuse. The chapters encompassing problems encountered by women of reproductive age and older provide a practical approach to pelvic pain and masses seen in the emergency department. They also include the management of breast disorders, the treatment of pelvic disorders, the treatment of pelvic
inflammatory disease, and approaches to managing complications of gynaecological procedures.

This book has been well researched and carefully referenced with the casualty officer in mind, but would also be very helpful to the obstetric and gynaecology specialist registrar in training. I hope to see it lining the shelves when I next have to attend my accident and emergency department!

Reviewed by Andrew Horne, PhD, MRCOG Specialist Registrar in Obstetrics and Gynaecology, Edinburgh, UK 